\title{
Laboratory Science Training Improvement Using an Immunohematology Simulation Lab
}

\author{
Sonya Hidalgo \\ MAT, MLS(ASCP) \\ McNeese State University, College of Nursing and Health Professions \\ Box 91820. Lake Charles, LA, USA, 70609 \\ United States of America
}

\begin{abstract}
Medical Laboratory Scientists are certified professionals who conduct assays on body fluids and other biological samples. To work in this profession one must earn a Bachelor of Science degree and complete a 12-month hospital internship. In the current state, most Medical Laboratory Science students gain their hands-on portion of their experiences in hospital laboratories. This time is severely limited, however, and can lead to inexperience upon the recent graduate beginning work. This article explores a grant-financed project to enhance the Medical Laboratory Science students' Immunohematology experiences on campus prior to hospital experiences. The goals of this exposure are to reduce hospital errors and produce more marketable experienced graduates. This was accomplished through external grant funding, purchase of equipment and supplies, planning and management of a simulated immunohematology laboratory, and student participation in pre- and post-tests. This work was made possible through a Louisiana Board of Regents grant and the support of the College of Nursing and Health Professions at McNeese State University.
\end{abstract}

Keywords: medical, laboratory, immunohematology, grants, blood banking, stake holders

\section{INTRODUCTION}

The immune system is an interconnected system of cells, tissues, and organs that can mount responses against a variety of foreign particles. An antigen is any substance that, when introduced into an animal, causes the production of an antibody that reacts specifically with the antigen [1]. In the immunohematology department (commonly known as blood bank), scientists seek to identify relevant antibodies in patients to ensure safe blood transfusion practices [2].

The class of the antibody being produced by a patient is important, as it is IgG antibodies that often cause the greatest issue in transfusions [3]. In a clinical blood bank lab, different phases of in-vitro testing are carried out, to attempt to identify patient antibodies with as much specificity as possible. One such test is an antibody screen, which uses patient plasma and reagent red blood cells (RBCs) to determine presence or absence of clinically significant antibodies. Agglutination is considered to be a positive result, and mimics what could happen in vivo. Scientists must take any positive antibody screen and follow up with an antibody panel, which serves to identify the specific patient antibody that is present [1].

Medical laboratory scientists are trained to collect and analyze biological specimens such as urine, body fluids, tissue, blood, and other substances. As of a 2016 survey, there were over 335,000 individuals employed in this field nationwide [4]. An inexperienced hospital scientist can cause severe negative patient results in blood bank testing, as many of these tests culminate in the issuing of a "blood product"- a unit of RBCs or plasma that need to be 
compatible to the patient who is receiving them. Inexperienced blood bankers should be paired with a mentor or guide until full proficiency can be established. This can be difficult in a working environment, however, due to the fluctuations in workflow and limited hospital budget toward over-staffing. University-based programs geared toward this type of education can serve this field by presenting the best, most stringent training as possible to alleviate the burden of under-practiced inexperienced scientists. This study was designed to obtain and utilize grant funds to purchase blood-banking equipment not usually found in the university labs to better train the upcoming students and produce more experienced new graduate scientists.

\section{PROJECT GOALS AND OBJECTIVES}

The MLS program trains students for the first 3 years to be ready for their senior level offcampus hospital internship. This preparation did not include Blood Bank lab experiences. The student did not get practical Blood Bank lab experience until they reach the hospital level, and then it is only allotted for eight weeks. The students need more time to become proficient is such a crucial life-saving department. The Medical Laboratory Science degree offered by this institution prepares students for a national certification exam, the Board of Certification (BOC) exam, prepared by the American Society of Clinical Pathologists (ASCP). The pass-rate of the university's graduates is one of the elements evaluated during the department's inspection and accreditation process, performed by NAACLS (National Accrediting Agency for Clinical Laboratory Sciences). One of the primary goals of this project is to produce better-prepared MLS graduates, and this is assessed through their BOC scores. The Blood Bank subscore counts toward $20 \%$ of the final score, so improvement in the students Blood Bank knowledge and skills can directly influence our department's BOC pass rate and our accreditation rating.

The impact of this project should be directly observed by student performance in the oncampus Procedures Laboratory. The curriculum in this class should cover a spectrum of laboratory procedures for the Medical Laboratory Scientist. Currently this class covers serology, microbiology, and dilution techniques. Blood banking is only able to be taught in a mini-lab session, using a few of the reagents in inventory. Approval of this project would allow for a condensation of serology lab and creation of a Blood Bank lab rotation. This would positively affect the offerings in this course tremendously. The goals for this project were:

Goal 1: To establish junior level Blood Bank lab proficiency

○ Objective: Create a 4-week Blood Bank lab

- Measurement: The student will demonstrate a minimum of $75 \%$ proficiency when completing 10 specimen "unknowns" for Blood Bank lab assignments.

$>$ Goal 2: To enhance the students' knowledge of troubleshooting laboratory equipment

- Objective: The students will practice troubleshooting instrumentation using scenarios of equipment failure or reagent failure.

- Measurement: The student will demonstrate understanding (with a 75\% minimum grade) by diagnosing and fixing the source of the problem in a given scenario, and turning in their solution on a lab report.

Goal 3: To produce better prepared MLS graduates

o Objective: Provide better-prepared internship students for our affiliated hospital labs to train, and increase the pass rate of the graduates' BOC (Board of Certification) tests.

- Measurement: Feedback from the Blood Bank instructors in our off-campus hospital labs, and any increase in their satisfaction will be deemed as successful 


\section{Timeline of Project Implementation}

\section{PROJECT WORK PLAN}

Table 1 demonstrates the timeframe for implementation of this project. Evaluation of this program will be accomplished through pre-test and post-test scores, student evaluation of instruction scores, and hospital-based laboratory Blood Bank instructor feedback.

Table 1. Timetable of Project Implementation

\begin{tabular}{|l|l|l|}
\hline Time Frame & \multicolumn{1}{|c|}{ Activity } & \multicolumn{1}{|c|}{ Benchmarks } \\
\hline Summer & $\begin{array}{l}\text { Verify already existing reagent supply; } \\
\text { Request updated price quotes on } \\
\text { equipment and supplies }\end{array}$ & Receive updated prices \\
\hline Summer & Order equipment and supplies & $\begin{array}{l}\text { Paperwork processed through University } \\
\text { purchasing department }\end{array}$ \\
\hline Fall & $\begin{array}{l}\text { Update affected syllabi to reflect new lab } \\
\text { sessions }\end{array}$ & Approval of syllabi by department head \\
\hline Late Fall & $\begin{array}{l}\text { Receive shipments of equipment and } \\
\text { supplies }\end{array}$ & $\begin{array}{l}\text { Purchasing department confirms receipt of } \\
\text { shipment; department inventory is updated } \\
\text { to demonstrate receipt }\end{array}$ \\
\hline Winter & $\begin{array}{l}\text { Set up equipment, run simulations to } \\
\text { verify student workstations and supplies }\end{array}$ & $\begin{array}{l}\text { Successful practice session completed at } \\
\text { each workstation }\end{array}$ \\
\hline Early Spring & $\begin{array}{l}\text { Incorporate new labs into course } \\
\text { "Procedures Laboratory" }\end{array}$ & $\begin{array}{l}\text { Results of students assessments - lab } \\
\text { reports, SEIs }\end{array}$ \\
\hline Late Spring & $\begin{array}{l}\text { Evaluate feedback from off-campus Blood } \\
\text { Bank instructors pertaining to student } \\
\text { readiness after new labs }\end{array}$ & $\begin{array}{l}\text { Receive feedback and continue with } \\
\text { program, improving per suggestions and } \\
\text { feedback }\end{array}$ \\
\hline
\end{tabular}

\section{Impact on Curriculum and Instruction}

When teaching Blood Bank theory, pictures and videos must be utilized because of the lack of instrumentation available in our on-campus laboratory. With implementation of this equipment and supplies purchase, pedagogy will be adapted to expand Blood Bank beyond classroom teaching and into laboratory experiences. Research has clearly shown that handson activities for students allow them to learn the material to a higher level needed for critical thinking and problem solving [5].

The expansion of the students' laboratory experiences to include Blood Bank activities will allow for increasing complexity of the classroom Blood Bank curriculum, because of the higher level learning the students are already experiencing.

\section{Materials}

Equipment requested is based on the MLS laboratory student capacity of 24 student stations. To be conservative with the major equipment requested, we will utilize groups of three students. To simulate the hospital laboratory environment as well as possible, the other equipment and supplies requested are calculated to populate the 24 student stations.

Item 1: Medical Laboratory Science students are required to learn Blood Bank, in the classroom and the laboratory setting. The creation of an on-campus Blood Bank lab will allow the students the necessary time and practice needed to master this skill set. This equipment is essential because currently the MLS students do not get hands-on work in this subject until their senior off-campus labs, and this is limited to only 8 weeks. Eight weeks of experience working in the Blood Bank department is not sufficient for the level of expertise this critical work demands. This equipment is a good choice because even in the high-workload environment of the hospital labs, they last a long time (usually 20+ years) with minimal monthly preventative maintenance. 
Item \#2: The MLS lab does currently have standard test tube racks available for each student station; however, test tubes are larger than Blood Bank tubes. The Blood Bank test tubes slide sideways and sometimes fall over when placed in the regular racks. The requested Blood Bank racks are comparable but cheaper than the ones found in a hospital Blood Bank lab. These racks will allow the students' work in progress to be stored in the appropriate manner while they add sample and reagents. The racks will also keep the Blood Bank tubes from shifting sideways and spilling out the students' work.

Item \#3: The reagent racks are comparable to the ones found in a hospital lab, and will give the students practice at a hospital-like work station. The racks allow for organization and standardization of the Blood Bank reagents. The project's planned lab procedures will reinforce the classroom lessons of the importance of standardization of their steps. In a hospital, the Blood Bank department personnel performs critical life-saving work. If a work error or mistake happens, it can cost a patient their life. As a Medical Laboratory Scientist, I do take the responsibility of teaching this subject very seriously. These students are literally learning steps and skills to reduce human error and save patients' lives. By giving them the exposure to this subject in their labs here on campus, it will complement and add to the 8-week rotation they receive during their senior year off-campus.

Item \#4: The MLS lab is already equipped with microscopes for each student station. The microscopes are utilized for other MLS labs, including hematology (the study of blood cells), urinalysis (cells, bacteria, and other formed substances in urine), and microbiology (bacteria, parasites, and yeast). This request does not include additional microscopes; it seeks to utilize the microscopes already on hand and make them Blood Bank friendly. The viewers requested lie on the microscope stage, and serve as a channel for the Blood Bank tube (Item \#10) to fit in. One of the crucial steps the students must learn in Blood Bank is to check microscopically for cell agglutination (cells clumping together). Cell agglutination demonstrates a positive Blood Bank reaction, and can signal several different triggers in this department. In the Blood Bank classroom, the students learn about the different issues that can cause cell agglutination. This lab gives them the opportunity to actually see it in the microscope, and the viewers are essential for this step.

Item \#5: The test tube dispensers requested serve organizational and housekeeping purposes. The dispensers are used in the real hospital Blood Bank department, and allow for a safe way to store the open box of tubes (Item \#10, listed under supplies) to prevent spillage. Without the dispensers, the boxes will need to be set on the countertop for the students to reach in to remove tubes. The stations do not stay organized when equipment and supplies get left out on the countertop. The dispenser also serves as a dust protector for the open box of tubes. If dust gets inside of a tube, it can cause a false-agglutination (clumping) reaction.

Item \#6: The MLS lab has grounded electrical outlets on the lab tables. The surge protectors are requested in order to offer further protection for the electrical equipment (Item \#1). In the case of lightning strike or other power surge, it is prudent to utilize surge protectors in this way.

Item \#7: The heat block is a mandatory part of the test procedure that the students will be learning. It is sized for the Blood Bank tubes, and heats them to approximately body temperature, which is the basis for the test procedure. The heat block listed in this budget was picked because of the tube capacity- it will require only this one heat block to hold all of the students' work for one day. Smaller heat blocks may cost less individually, but we would need 
to purchase multiple blocks to have enough slots. Economically, one heat block with this size capacity is the better choice.

Item \#8: Blood Bank testing utilizes saline, and the 8 boxes requested would be a one-time purchase to coordinate with the 8 Cell Washers (Item \#1). Based on the testing procedures, each set of tests use approximately 40 milliliters of saline. Each box holds 5 gallons of saline, so each box should last for approximately 473 test cycles (about 2 years). At that point the boxes and tubing will be retained, and the boxes will be refilled with bulk bought saline from the department.

Item \#9: The holders requested are fitted to hold the Blood Bank Boxed Saline (Item \#8). The MLS lab has a defined amount of tabletop space, and student stations already have microscopes and other lab supplies kept there. In addition, the students usually have their notes and notebooks out while performing tests. The purpose of these holders is to make the most efficient space possible, keep the students' stations organized, and mimic the style of the actual hospital Blood Bank labs.

Item \#10: The packs of test tubes are standard for Blood Bank labs, and each pack contains 250 tubes. For the set of testing planned for the student labs, one pack should last one semester per student. For the 24 available student stations, the estimated consumption will be 24 packs of tubes per semester. This budget includes one case per station (cheaper due to bulk pricing), and is expected to last 8 years. Table 2 demonstrates that itemized budget for this project.

Table 2. Itemized Budget

\begin{tabular}{|l|l|c|c|c|}
\hline & Equipment & Unit Price & Quantity & Total \\
\hline Item \#1 & Helmer UltraCW Automatic Cell Washer & $\$ 9054.29$ & 8 & $\$ 72,434.32$ \\
\hline Item \#2 & Blood Bank Specimen Racks & $\$ 140.00$ & 24 & $\$ 3360.00$ \\
\hline Item \#3 & Blood Bank Reagent Racks & $\$ 75.00$ & 24 & $\$ 1800.00$ \\
\hline Item \#4 & Microscope Test Tube Viewers & $\$ 12.50$ & 24 & $\$ 300.00$ \\
\hline Item \#5 & Test Tube Dispensers & $\$ 65.00$ & 24 & $\$ 1560.00$ \\
\hline Item \#6 & Surge Protectors & $\$ 68.99$ & 8 & $\$ 551.92$ \\
\hline Item \#7 & Heat Block & $\$ 1107.90$ & 1 & $\$ 1107.90$ \\
\hline Item \#8 & Boxed Blood Bank Saline & $\$ 40.30$ & 8 & $\$ 322.40$ \\
\hline Item \#9 & Boxed Blood Bank Saline Holders & $\$ 216.00$ & 8 & $\$ 1728.00$ \\
\hline Item \#10 & Packs of Test Tubes & $\$ 57.60$ & 24 & $\$ 1382.40$ \\
\hline
\end{tabular}

\section{PROJECT EVALUATION}

Feedback from the hospital-based Blood Bank instructors will be used to adjust the curriculum as needed. For all of the evaluations listed below, the student must achieve a $75 \%$ (the minimum grade that is passing in the MLS degree plan) to be considered as successful. Assessment of the goals for this project are:

$>\quad$ Goal 1: To establish junior level Blood Bank lab proficiency

- Activities: use the requested equipment to create a 4-week Blood Bank lab during the already existing MLS Procedures Lab.

○ Benchmark: Student will be able to complete 10 of each of the following: ABO/Rh, Antibody Screens, Antibody IDs

- Evaluation- The instructor will provide student 10 "unknowns", the student will process each from beginning to end and write up a lab report with the results.

- Benchmarks: Student will be able to progress each week and learn the steps and purposes of the above listed tests and the required reagents. 
- Evaluation: Successful completion of each week of lab activities, including reagents, equipment, and testing performed. Students will gain credit for this work by turning in lab reports.

Goal 2: To enhance the students' knowledge of troubleshooting laboratory equipment

○ Activities: During week 4 of the proposed timeline, the students will be provided with scenarios of equipment failure and must diagnose and fix the issue. The students will also be presented with a reagent failure and must diagnose the problem and propose a resolution.

- Benchmarks: Student will be able to recognize a problem, diagnose the source, fix the problem.

- Evaluation: Successful completion of Week 4 lab activity documented by achieving the above mentioned minimum grade $(75 \%)$ on the lab report.

Goal 3: To produce better prepared MLS graduates

- Activities: Creation of Blood Bank lab rotations on-campus, before the senior level hospital rotations. Students will complete labs as explained above.

- Benchmarks: Student success rate on the national certification exam Board of Certification (BOC) test, specifically Blood Bank subscore

- Evaluation: Inquire for feedback from hospital-based Blood Bank instructors for evaluation of student preparedness, compared to prior system.

\section{Stakeholder Engagement}

A stakeholder is defined as a person or group that has a definable interest or concern in a specific program or organization [6]. An expanded definition can encompass anyone with the "potential to influence or who may be influenced by particular actions or aims" [7]. Stakeholders can be further delineated as needed for the survival of an organization [8]. When one is planning a project or program, identifying the interested parties could be crucial for potential success and implementation. Stakeholder involvement is critical, as it is documented that this engagement increases community buy-in and improves the quality of the decisionmaking process [9]. Stakeholders for this project include local hospitals and industry and the field of nursing.

\section{Relationships with Industrial /Institutional Stakeholders}

This project will strengthen our already strong relationship with our hospital-affiliated laboratories, who train our students for their senior internship rotations. By providing them with better trained interns, they will be more satisfied with the students they receive and train. Also, by seeking their feedback on this program, they will know that they are valuable teammembers even though they are off-campus. The MLS department maintains a strong professional relationship with our local hospital laboratories, and this added component will reinforce our commitment of providing high quality students. This proposal will also strengthen these connections for future sources of external funding, as they continue to supply us with their expired reagents and know how these reagents are being utilized. The ability to orient and train students for a year, and thereby shortening their training period after hire, is a positive impact directly upon the hospital laboratories' availability of new hires to contribute in the workforce [10]. It is in the hospitals' best interests to maintain strong relationships with the local university, as it provides future workforce human resources [11].

This project will benefit academic resources in Louisiana because the majority of our MLS students are from this state and often plan to stay in this state. We are training our own future workforce, and it is mutually beneficial to train them to the best capacity possible. The current MLS students are Louisiana's future MLS professionals, performing life-saving crossmatches in our local hospitals. Our area is expecting population growth, and the demand on our hospitals 
will be greater than ever. The entire Lake Charles area is experiencing a large industrial sector growth, with thousands of temporary and permanent jobs expected. Sasol manufacturing, Louisiana Natural Gas (Cameron LNG), Chennault International Airport Complex, and ShawWestinghouse are only a few of the industrial companies currently expanding in this area (Southwest Louisiana Chamber Alliance, n.d.). As the local population grows, the demand on our local hospitals' workforce will continue to increase. We need to raise the academic bar now to begin turning out the highest level graduates possible.

\section{Relationships with Nursing and Other Healthcare Stakeholders}

Within the hospital environment, it is traditionally nursing service's role to administer blood transfusions [13]. Physicians, healthcare providers, and nurses rely upon the accuracy of MLS professionals to ensure safe products for transfusions. Without reciprocal trust, healthcare practices can be delayed causing negative patient outcomes. The importance of nursing service and allied health departments, including the laboratory, maintaining positive professional relationships cannot be overstated [14]. Nurses are responsible for interpreting and carrying out physicians' medical orders, but without a working knowledge of laboratory basics he or she is at a large disadvantage. Negative relationships between these departments can severely impact patient care [15].

\section{RESULTS}

Four years into this study, the pre-test and post-test scores have shown dramatic differences. In the 2016 session, pre-test knowledge scores had a class average of $20 \%$, with post-test scores demonstrating a 95\%. In 2017: pre-test average was 15\%, post-test class average was 100\%. 2018 and 2019 demonstrated an increase in student learning of greater than $90 \%$. Informal assessments were carried out with clinical instructors, including verbal interviews and email correspondence. Feedback surveys from the hospital clinical instructors were obtained, and demonstrated a $72 \%$ increase in satisfaction levels toward their new students. Additionally, student lab reports and participation levels met all of the defined benchmarks. This program was deemed successful based on the criteria defined in the beginning of the study.

\section{CONCLUSION}

In a high-demand field such as Medical Laboratory Science, hands-on learning is the best means to aid the student in understanding the complex material. This project added hands-on Immunohematology equipment to the junior-level curriculum that was not available prior to this project. Student improvement has been noted all four years since its inception, and the project will continue in the future. Immunohematology is a complex department requiring the laboratory professional to critically-think and troubleshoot on a daily basis. This project has raised the educational opportunities and marketability of the university's medical laboratory science students and graduates.

\section{References}

Howard, P. (2017). Basic \& applied concepts of blood banking and transfusion practices (4th ed.). St. Louis, MO: Elsevier.

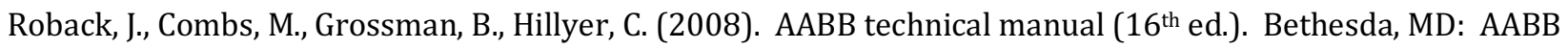
Publishing

Stevens, C. (2010). Clinical immunology \& serology: A laboratory perspective (3 ${ }^{\text {rd }}$ ed.). Philadelphia, PA: F.A.Davis Company.

Medical and clinical laboratory technologists and technicians. (2018). Retrieved from https://www.bls.gov/ooh/healthcare/medical-and-clinical-laboratory-technologists-and-technicians.htm 
American Chemical Society (2015). Importance of hands-on laboratory science. Retrieved from: https://www.acs.org/content/acs/en/policy/publicpolicies/education/computersimulations.html

Business Dictionary. (n.d.). Stakeholder. Retrieved from http://www.businessdictionary.com/definition/stakeholder.html

Ekirapa-Kiracho, E., Ghosh, U., Brahmachari, R., \& Paina, L. (2017). Engaging stakeholders: Lessons from the use of participatory tools for improving maternal and child care health services. Health Research Policy and Systems, 15, 17-28. https://doi.org/10.1186/s12961-017-0271-z

Freeman, R. E., \& Reed, D. (1983). Stockholders and stakeholders: A new perspective on corporate governance. California Management Review. https://doi.org/10.2307/41165018

Chanse, V. (2016). Engaging stakeholders in the sea level rise design process: A pilot project on Maryland's eastern shore. The International Journal of Climate Change: Impacts and Responses, 8(3), 81-88. Retrieved from https://www.on-climate.com

Barnow, B. S., \& Spaulding, S. (2015). Employer involvement in workforce programs: What do we know? In C. Van Horn, T. Edwards, \& T. Greene (Eds.), Transforming U.S. workforce development policies for the 21st century (pp. 231-264).

Spaulding, S., \& Martin-Caughey, A. (2015, December). The goals and dimensions of employer engagement in workforce development programs. Urban Institute, 1-15. Retrieved from https://www.urban.org/

Southwest Louisiana Chamber Allliance (SWLA). (n.d.). About SWLA growth. Retrieved from: https://www.allianceswla.org/index.php

Bielefeldt, S., DeWitt, J. (2010). The rules of transfusion: Best practices for blood product administration. American Nurse Today, 5 (4). Retrieved from: https://www.americannursetoday.com/the-rules-of-transfusionbest-practices-for-blood-product-administration-2/

Ewertsson, M., Allvin, R., Holmström, I. K., \& Blomberg, K. (2015). Walking the bridge: Nursing students' learning in clinical skill laboratories. Nurse Education in Practice, 15, 277-283.

https://doi.org/10.1016/j.nepr.2015.03.006

Passiment, E., Meisel, J. L., Fontanesi, J., Fritsma, G., Aleryani, S., \& Marques, M. (n.d.). Decoding Laboratory Test Names: A Major Challenge to Appropriate Patient Care. Journal of General Internal Medicine, 28(3), 453-458. Retrieved from https://mcneese.idm.oclc.org/login?url=http://search.ebscohost.com/login.aspx?direct=true\&db=boh\&AN=BAC D201400133940\&site=eds-live 